BNL -64510

Informal Report

\title{
Fast Polynomial Approach to Calculating Wake Fields
}

\author{
Charles I. Goldstein and Ronald F. Peierls * \\ Department of Applied Science \\ Brookhaven National Laboratory
}

June 15,1997

\section{Introduction}

In the computation of transverse wake field effects in accelerators [1] it is necessary to compute expressions of the form

$$
\Delta x_{i}^{\prime}=\frac{N_{b} Z^{2} e^{2}}{A m_{u} c^{2} \gamma} \frac{1}{N_{m}} \sum_{j=1}^{N_{m}} V_{1}\left(z_{j}-z_{i}\right) x_{j}
$$

where $\left(x_{i}, z_{i}\right)$ represent the transverse and longitudinal coordinates of the $i$-th of $N_{m}$ macroparticles, and $V_{1}(z)$ is a potential, which vanishes except when $z$ is positive. All other symbols represent constants. The prime denotes differentiation w.r.t. longitudinal distance.

It is usually desired to compute this a large number of times, the values of $z_{i}$ and $x_{i}$ being different at each iteration, other quantities remaining the same. The problem in practical applications is that the computational work grows as $N_{m}^{2}$. Thus even using parallel computation to achieve speedup, the elapsed time to obtain a result still increases linearly with $N_{m}$.

We introduce here an approximate method of evaluating the sum in (1) whose computational work increases only as $N_{m} \log N_{m}$. It involves some significant initial computation which does not have to be repeated at each

"Work supported by the U.S. Department of Energy Office of Computational and Technology Research under contract DE-AC02-76CH00016. 
subsequent iteration. The basis of the approach is to replace the individual contributions of a group of distant macroparticles with a local series expansion. In this respect it is similar in spirit to the so called fast multipole method [2].

\section{Outline of the method.}

To simplify, the discussion, we write it as a more generic problem, that of computing

$$
a\left(z_{i}\right)=\sum_{j} x_{j} \phi\left(z_{j}-z_{i}\right), \quad 1 \leq i, j, \leq N_{m}
$$

where $\phi(z)$ vanishes for $z \leq 0$ and $z_{i}$ are normalized so that $0 \leq z_{i} \leq 1$.

We consider a decomposition of [0.1] into $M$ subintervals (bins) $I_{\beta}, M>\beta \geq 0$, by choosing points

$$
0=Y_{M}<Y_{M-1}<\cdots<Y_{0}=1
$$

the midpoint and halfwidth of the interval $I_{\beta}$ being specified as

$$
\bar{z}_{\beta}=\frac{\left(Y_{\beta}+Y_{\beta+1}\right)}{2}, \quad h_{\beta}=\frac{Y_{\beta}-Y_{\beta+1}}{2},
$$

respectively. We do not require the decomposition to be uniform. Each point $z_{i}$ lies in a unique bin labelled by the index ${ }^{1} \beta_{i}$. We can then rewrite (2) as:

$$
a\left(z_{i}\right)=\sum_{\beta \leq \beta_{i}} \sum_{z_{j} \in I_{\beta}} x_{j} \phi\left(z_{j}-z_{i}\right)
$$

The method we adopt is to approximate $\phi$ as a truncated double power series in the offsets of $z_{i}$ and $z_{j}$ from the centers of the bins in which they lie. A different expansion is needed for each term in the sum over $\beta$. In general, however, the smaller each interval the fewer expansion terms will be needed. Thus there must be a careful tradeoff between keeping the number of bins small, without letting the order of the expansion grow too much.

The expansion coefficients must be recalculated at each iteration, with a procedure which is linear in $N_{m}$. Thus the overall complexity is of order

\footnotetext{
${ }^{1}$ Where there is no ambiguity, we sometimes refer to the interval $I_{\beta}$ as the bin $\beta$.
} 


\section{DISCLAMMERR}

Portions of this document may be illegible in electronic image produets. Images are produced from the best available original document. 
$M \cdot N_{m}$. We develop a non-uniform decomposition procedure. such that $M$ increases as $\log N_{m}$, so that the overall scaling behavior is $N_{m} \log N_{m}$. Of course. improvement in scaling behavior is of no advantage if the computations required to generate the coefficients are much more complex than those in the direct calculation: the proposed algorithm is designed to reduce the complexity of those parts of the calculation which must be repeated at each iteration.

\section{Power series formulation}

For any point $z \in[0,1]$, we can define the offset $\delta(z)$ from the midpoint of the interval $\beta(z)$ in which it lies:

$$
z=\bar{z}_{\beta(z)}+\delta(z)
$$

We also define the separation between the midpoints of tro bins:

$$
\Delta_{3 \beta^{\prime}}=\bar{z}_{\beta^{\prime}}-\bar{z}_{\beta}
$$

Let $z, z^{\prime}$ be arbitrary points lying in $I_{\beta}, I_{\beta}$, respectively. Defining

$$
\delta_{z z^{\prime}}=\delta\left(z^{\prime}\right)-\delta(z)
$$

we can then express the separation:

$$
z^{\prime}-z=\delta_{z z^{\prime}}+\Delta_{\beta \beta^{\prime}}
$$

We say that the bin $\beta^{\prime}$ is consistent with $\beta$ to order $P$ if for all $z \in I_{\beta}, z^{\prime} \in I_{\beta^{\prime}}$ we can approximate $\phi\left(z^{\prime}-z\right)$ by a finite power series:

$$
\begin{aligned}
\phi\left(z^{\prime}-z\right) & =\phi\left(\delta_{z z^{\prime}}+\Delta_{\beta \beta^{\prime}}\right) \\
& \approx \sum_{p=0}^{p} A_{\beta \beta^{\prime}}^{(p)} \quad \delta_{z z^{\prime}}^{p} .
\end{aligned}
$$

Since $\phi(z)$ vanishes identically for all $z \leq 0$, the function $\phi\left(z^{\prime}-z\right)$ considered as an analytic function of $\delta_{z z^{\prime}}$ has a singularity at $\delta_{z z^{\prime}}=-\Delta_{\beta \beta^{\prime}}$. Thus the power series expansion in $\delta_{z z^{\prime}}$ has a radius of convergence $\Delta_{\beta \beta^{\prime}}$, so that convergence of the power series expansion is likely to deteriorate as $\left|\delta_{z z^{\prime}}\right|_{\max }=h_{\beta^{\prime}}+h_{\beta}$ approaches $\Delta_{\beta \beta^{\prime}}$. From this we see that the approximation is not valid in general when $z, z^{\prime}$ are in the same bin or immediately adjacent bins. Define $\beta^{(0)}$ to be the first bin consistent with $\beta\left(\beta^{(0)}<\beta-1\right)$. 
If we carry out the binomial expansion of $\delta_{z z^{\prime}}=\left[\delta\left(z^{\prime}\right)-\delta(z)\right]^{p}$ we obtain

$$
\phi\left(z^{\prime}-z\right)=\sum_{q=0}^{P} \sum_{r=0}^{P-q} \frac{(q+r) !}{q ! r !} A_{33^{\prime}}^{(q+r)}[-\delta(z)]^{q} \delta\left(z^{\prime}\right)^{r}
$$

Using this expansion and rearranging terms, we get

$$
\begin{aligned}
a\left(z_{i}\right) & =a^{(0)}\left(z_{i}\right)+a^{+}\left(z_{i}\right), \\
a^{(0)}\left(z_{i}\right) & =\sum_{\beta_{i} \geq \beta_{j}>\beta_{i}^{(0)}} \sum_{z_{j} \in I_{\beta_{j}}} x_{j} \phi\left(z_{j}-z_{i}\right) \\
a^{+}\left(z_{i}\right) & =\sum_{q=0}^{P} \delta\left(z_{i}\right)^{q} \sum_{\beta_{j} \leq \beta_{i}^{(0)}} \sum_{r=0}^{P-q} \sum_{z_{j} \in I_{\beta_{j}}} x_{j} \delta\left(z_{j}\right)^{r}(-1)^{q} \frac{(q+r) !}{q ! r !} A_{\beta_{i} \beta_{j}}^{(q+r)}
\end{aligned}
$$

We define two coefficients:

$$
\begin{aligned}
\alpha_{r}^{\beta} & =\sum_{z j \in I_{\beta}} x_{j} \dot{\delta}\left(z_{j}\right)^{r} \\
C_{q \beta} & =\sum_{\beta^{\prime} \leq \beta^{(0)}} \sum_{r=0}^{P-q}(-1)^{q} \alpha_{r}^{\beta^{\prime}} \frac{(q+r) !}{q ! r !} A_{\beta \beta^{\prime}}^{(q+r)}
\end{aligned}
$$

and obtain, finally:

$$
a\left(z_{i}\right)=a^{(0)}\left(z_{i}\right)+\sum_{q=0}^{P} C_{q \beta_{i}} \delta\left(z_{i}\right)^{q}
$$

The key to speeding up the calculation is to precompute the quantities $A_{\beta \beta^{\prime}}^{(p)}$ and $\alpha_{r}^{\beta}$ as early as possible, and to keep $P$ and the number of distinct intervals $I_{\beta_{j}}$ as low as possible. The coefficients $A$ depend only on the function $\phi$ and the decomposition. Different $z_{i}$ may require different decompositions. However, provided all decompositions which will be used are known in advance, the coefficients only need to be calculated once (at the beginning of the computation). The coefficients $\alpha$ depend on the specific values of the $\left\{z_{i}\right\}$, and must be updated for each decomposition used for each iteration. For a given decomposition, the task is linear in $N_{m}$. Although the number of different decompositions used at each iteration increases with $N_{m}$, we shall show how they can be organized hierarchically in such a way as to preserve the $N_{m} \log \left(N_{m}\right)$ dependence.

The number of terms required in the expansion (5), $P$, will depend on $\beta$ and $\beta^{\prime}$. 


\section{Decomposition}

Consider a particular $z_{i}$ in eq (2), and suppose we wish to fix $P$ at some predetermined limit. Then, depending on the particular expansion used in equation (5), we could assign $Y_{1}$ in (3) to be the smallest value of $z$ such that the expansion (5) truncated at $P$ is of acceptable accuracy for all $Y_{1} \leq z_{j} \leq 1$. We then define $Y_{2}$ such that the expansion is sufficiently accurate for $Y_{2} \leq z_{j} \leq Y_{1}$, etc.

To carry out this analysis for each $z_{i}$, however, would defeat the concept of precalculating most of the coefficients. Instead, we start with a fixed decomposition into a large number of equal intervals and build up the actual bins $\beta$ by combining a number of adjacent low level intervals.

We divide the interval $[0,1]$ into subintervals by recursive bisection. At the $l$-th stage we define $2^{l}$ subintervals

$$
I(l, k)=\left[Z_{k+1}^{l}, Z_{k}^{l}\right], \quad 0 \leq k<2^{l}: \quad Z_{k}^{l}=\left(2^{l}-k\right) \cdot 2^{-l}
$$

At level $l+1$ the interval $I(l, k)$ at level $l$ is partitioned into the two intervals $I(l+1.2 k)$ and $I(l+1,2 k+1)$.

Let $L$ be the deepest level of subdivision. (See Figure 1.) The point $z_{i}$ lies in some subinterval $I\left(L, k_{i}\right)$ at this level, which is defined to be the bin $\beta_{i}$. The remaining bins are defined, starting from the right hand boundary, at each step choosing the largest subinterval (i.e. the one at the lowest level) adjacent to the last one chosen, which is consistent with $\beta_{i}$ in the sense defined above, that is, separated from $I_{\beta_{i}}$ by a large enough gap to ensure that the truncated power series expansion is sufficiently accurate.

The precise specification is the following:

1. Choose $I_{0}=I\left(l_{0}, 0\right)$ where $l_{0}$ is the smallest value of $l$ for which this interval is consistent with $I_{\beta_{i}}$.

\section{Choose}

$$
I_{n}=I\left(l_{n}, k_{n}\right) ; \quad l_{n}=l_{n-1}+\lambda_{n}, k_{n}=2^{\lambda_{n}}\left(k_{n-1}+1\right)
$$

where $\lambda_{n}$ is chosen as the smallest value resulting in an interval consistent with $I_{\beta_{i}}$.

3. If no such $\lambda_{n}$ exists (i.e. if even the next subinterval at level $L$ is too close to $I_{\beta_{i}}$ ), then use the above formula with $\lambda_{n}=L-l_{n-1}$ and assign the previous interval, $I_{n-1}$, to be $\beta_{i}^{(0)}$. 
4. The remaining bins are all those at level $L$ between $\beta_{i}$ and $\beta_{i}^{(0)}$.

In the numerical examples reported in this paper, the criterion adopted is that at least one bin at the same level as $\beta^{\prime}$ must lie between it and $\beta$ for it to be consistent. This guarantees that $\left|\delta_{z z^{\prime}}\right| / \Delta_{3 \beta^{\prime}} \leq 0.5$.

The sums over $j$ in the definition of the $\alpha_{q}^{\beta}$ need only be carried out for the finest level. If $\beta_{0}, \beta_{1}$ are the two children of bin $\beta$ at some level, then we can write

$$
\begin{aligned}
\alpha_{q}^{\beta}= & \sum_{z_{j} \in I_{\beta}} x_{j}\left(z_{j}-\bar{z}_{\beta}\right)^{q} \\
= & \sum_{z_{j} \in I_{\beta_{0}}} x_{j}\left(z_{j}-\bar{z}_{\beta_{0}}-\frac{h_{\beta}}{2}\right)^{q}+ \\
& \sum_{z_{j} \in I_{\hat{\beta}_{1}}} x_{j}\left(z_{j}-\bar{z}_{\beta_{1}}+\frac{h_{3}}{2}\right)^{q} \\
= & \sum_{r=0}^{q} \Gamma_{r q}\left((-1)^{q-r} \alpha_{r}^{3_{0}}+\alpha_{r}^{\beta_{1}}\right)
\end{aligned}
$$

where

$$
\Gamma_{r q}=\frac{q !}{r !(q-r) !}\left(\frac{h_{\beta}}{2}\right)^{q-r}
$$

The extra work of calculating on all the coarser bins is of order $P^{2} M \log N_{m}$. In general, the functions $\phi$ with which we will be concerned are relatively smooth at large distances, so that the intervals $I_{n}$ will tend to increase in size as $n$ decreases.

\section{Specific Expansions}

In this paper we consider in detail two choices for the series expansion: Taylor Series and Chebychev expansions. The simplest choice is the Taylor series expansion. Its major disadvantage is the need to evaluate derivatives of the function $\phi$. If $\phi$ is defined in terms of observations, and no analytical form exists, then it may be difficult to evaluate the derivatives accurately. Even when the analytical form is known, evaluation of higher derivatives may be costly. 
The Chebyshev expansion requires only knowledge of the function itself, not of its derivatives. An additional advantage is that its accuracy is uniform orer the full expansion range. However, for simple analytical forms its extra complexity may not be worth while.

\subsection{Taylor Series}

One choice for the series expansion of $\phi\left(z^{\prime}-z\right)$ is to carry out a Taylor expansion about the point $\Delta_{\beta \beta^{\prime}}$. Specifically:

$$
\begin{aligned}
\phi\left(z^{\prime}-z\right) & =\phi\left(\delta_{z z^{\prime}}+\Delta_{\beta \beta^{\prime}}\right) \\
& =\sum_{p=0}^{\infty} \frac{\phi^{(p)}\left(\Delta_{33^{\prime}}\right)}{p !} \delta_{z z^{\prime}}^{p}
\end{aligned}
$$

where

$$
\phi^{(p)}(z) \equiv \frac{\partial^{p} \dot{\varphi}(z)}{\partial z^{p}}
$$

Hence in equation (8) we can make the assignment

$$
A_{\beta \beta^{\prime}}^{(q+r)}=\frac{\mathcal{o}^{(q+r)}\left(\Delta_{33^{\prime}}\right)}{(q+r) !}
$$

\subsection{Chebyshev Expansion}

An alternative approach is to use Chebyshev polynomials. We observe that

$$
-h_{\beta}-h_{\beta^{\prime}} \leq \delta_{z z^{\prime}} \leq h_{\beta}+h_{\beta^{\prime}}
$$

Defining $w=h_{\beta}+h_{\beta^{\prime}}, Y=\delta_{z z^{\prime}} / w$ (so that $Y \in[-1,1]$ ) the expansion (5) becomes

$$
\phi(w Y+\Delta) \approx \sum_{p=0}^{P} A^{(p)}(w Y)^{p}
$$

where we have omitted the subscripts on $\Delta$ and $A^{(p)}$ for clarity. Defining $g(Y) \equiv \phi(w Y+\Delta)$, we expand $g(Y)$ in terms of Chebyshev functions $T_{k}(Y)$ on $[-1,1][3]$, with $N$ chosen large enough to provide the desired accuracy:

$$
g(Y) \approx \sum_{k=0}^{N-1} c_{k} T_{k}(Y)-\frac{1}{2} c_{0}
$$


The Chebyshev coefficients, $c_{k}$, are given by

$$
c_{j}=\frac{2}{N} \sum_{k=0}^{N-1} g\left(\cos \left(\frac{\pi\left(k+\frac{1}{2}\right)}{N}\right)\right) \cos \left(\frac{\pi j\left(k+\frac{1}{2}\right)}{N}\right) .
$$

We next choose $P \leq N-1$ such that $c_{P+1}$ is sufficiently small (depending on the desired accuracy), and define our approximation to $g(Y)$ by

$$
g_{P}(Y)=\sum_{p=0}^{P} c_{p} T_{p}(Y)-\frac{1}{2} c_{0}
$$

Collecting terms, this can be rearranged as a polynomial in $Y$,

$$
g_{P}(Y)=\sum_{k=0}^{P} d_{p} Y^{p}
$$

where the coefficients $\left\{d_{k}\right\}$ can be computed from the $\left\{c_{k}\right\}$ using Clenshaw's recurrence [3]. Comparing with equation (12), we obtain the assignment

$$
A_{\beta 3^{\prime}}^{(p)}=\frac{d_{p}^{\beta \beta^{\prime}}}{\left(h_{3}+h_{\beta^{\prime}}\right)^{p}}
$$

where the superscript $\beta \beta^{\prime}$ has been added to the coefficients $d_{p}$ to reflect their dependence, through the definition of $g(Y)$, on $\Delta_{\beta \beta^{\prime}}$.

\subsection{Results}

The algorithm described here was implemented and tested for both Taylor and Chebyshev expansions. The potential used for the test example was

$$
V_{1}(z)= \begin{cases}\frac{z}{1+\alpha z^{3 / 2}} & \text { for } z>0 \\ 0 & \text { for } z \leq 0\end{cases}
$$

$\Delta x_{i}^{\prime}$, defined by (1), was evaluated at $N_{m}$ points, $\left(x_{i}, z_{i}\right)$, using direct calculation, as well as the algorithm described here with both Taylor and Chebyshev expansions. Each case was run for 1000 iterations, with $z_{i}$ chosen randomly on the interval $[0,1]$ for each iteration. For the results presented in Table I, we set $\alpha=.1$, and set each $x_{j}$ and all physical constants in (1) equal to one. The computations were performed using double precision floating 
point arithmetic on an Silicon Graphics Indigo 2 processor and were run for 1000 iterations. For each case the optimum number of bisection levels was chosen (i.e. the number which led to the shortest computation time).

As expected, the values obtained using truncated series expansions with $P+1$ terms converge to the value obtained by direct computation as $P$ increases. The first column of Table I contains the number of macroparticles, $N_{m}$, the second column contains the time required to compute $\Delta x_{i}^{\prime}$ for all $N_{m}$ macroparticles for 1000 iterations using direct calculation. The remaining columns give the corresponding times for both Taylor and Chebyshev expansions using different truncation orders $P$.

\begin{tabular}{||r|r|r|r|r||}
\hline$N_{m}$ & $T_{\text {direct }}$ & $P$ & $T_{\text {Taylor }}$ & $T_{\text {Chebyshev }}$ \\
\hline \hline 20 & 1.7 & 1 & $.6(4)$ & $.6(4)$ \\
& & 2 & $.8(4)$ & $.7(4)$ \\
& & 3 & $.8(3)$ & $.8(3)$ \\
& & 4 & $.9(3)$ & $.9(3)$ \\
\hline 50 & 10.5 & 1 & $1.9(5)$ & $1.8(5)$ \\
& & 2 & $2.5(5)$ & $2.2(5)$ \\
& & 3 & $2.7(5)$ & $2.6(5)$ \\
& & 4 & $3.0(4)$ & $3.1(5)$ \\
\hline 100 & 41 & 1 & $4.3(6)$ & $4.1(6)$ \\
& & 2 & $5.3(6)$ & $5.0(6)$ \\
& & 3 & $6.3(6)$ & $6.1(6)$ \\
& & 4 & $6.7(5)$ & $6.6(5)$ \\
\hline 200 & 166 & 1 & $9.3(7)$ & $8.9(7)$ \\
& & 2 & $11.7(7)$ & $11.8(6)$ \\
& & 3 & $13.7(6)$ & $13.2(6)$ \\
& & 4 & $15.2(6)$ & $14.7(6)$ \\
\hline 500 & 1050 & 1 & $26(8)$ & $25(8)$ \\
& & 2 & $32(8)$ & $32(8)$ \\
& & 3 & $38(7)$ & $39(7)$ \\
& & 4 & $42(7)$ & $43(7)$ \\
\hline 1000 & 4200 & 1 & $56(9)$ & $55(9)$ \\
& & 2 & $69(9)$ & $69(9)$ \\
& & 3 & $80(8)$ & $86(9)$ \\
& & 4 & $89(8)$ & $90(8)$ \\
\hline \hline
\end{tabular}

Table I: Timing results for the direct calculation and proposed algorithm. All times are in seconds. The numbers in parentheses indicate the optimal 
number of recursive bisections used to define the lowest level bins.

First of all. Table I illustrates the $O\left(\mathrm{~N}^{-2}\right)$ increase in computing time with the number of macroparticles using the direct method. On the other hand, as indicated in the preceding discussion, the computational complexity of our proposed algorithm is of order

$$
T=O\left(k^{2} M^{\prime}\right)+O\left(P N_{m}\right)+O\left(p^{2} M^{\prime} \log N_{m}\right)
$$

where $k$ is the number of macroparticles per lowest level bin, and $M^{\prime}=$ $N_{m} / k$ is the number of lowest level bins. The first term on the right hand side coresponds to the computation of $a^{(0)}\left(z_{i}\right)$ (see eq $(6)$ ) over all particles, the second term corresponds to computation of the $\alpha_{r}^{\beta}$ over all lowest level bins (see eq(7)), and the last term corresponds to the computation of $\alpha_{q}^{\beta}$ over all the coarser bins. This last term accounts for the slightly faster than linear growth of computing time with $\gamma_{m}$ observed in Table I. Also note from eq(16) that to optimize $T, k$ must be chosen to balance the first and last terms on the right. The results in Table I indicate that, with the $V_{1}$ defined in eq(15), the optimal cost for small $P$ is achieved for $k$ between one and two. As $P$ increases, the choice of $k$ which optimizes the time also increases, so that $M^{\prime}$ decreases. For $P=4$ the optimal value of $k$ is between two and four.

We also note that the results using Taylor and Chebyshev expansions are almost identical, the computational cost using either becoming substantially less than that for the direct algorithm as $N_{m}$ increases. Analogous results were obtained using other potential functions $V_{1}$ whose values and derivatives could be computed quickly. If the computation of $V_{1}$ and its derivatives were to take a very long time, the optimal value of $k$ would be smaller. Furthermore, the Chebyshev expansion would be expected to be superior to the Taylor expansion in such cases.

The choice of $P$ depends on the desired accuracy. We have found from numerical experiments that, for $P=1$ the relative pointwise error is less than $10^{-2}$. For $P=2$ and $P=4$ this relative error is less tha $10^{-4}$ and $10^{-6}$, respectively, for boththe Taylor and Chebyshev expansions.

\section{References}

[1] S. Peggs and V. Mane, KRAKEN, A Numerical Model of RHIC Impedances, BNL 61166 
[2] L.Greengard and V. Rokhlin A Fast Algorithm for Particle Simulations, J.Comp. Phys. 73,2 (1987)

[3] W.H.Press, S.A.Teukolsky, W.T.Vetterling, and Brian P.Flannery, Numerical Recipes in $C$ (Second Edition), Cambridge University Press, Cambridge (1992).

\section{DISCLAIMER}

This report was prepared as an account of work sponsored by an agency of the United States Government. Neither the United States Government nor any agency thereof, nor any of their empioyees, makes any warranty, express or implied, or assumes any legal liability or responsibility for the accuracy, completeness, or usefulness of any information, apparatus, product, or process disclosed, or represents that its use would not infringe privately owned rights. Reference herein to any specific commercial product, process, or service by trade name, trademark, manufacturer, or otherwise does not necessarily constitute or imply its endorsement, recommendation, or favoring by the United States Government or any agency thereof. The views and opinions of authors expressed herein do not necessarily state or reflect those of the United States Government or any agency thereof. 

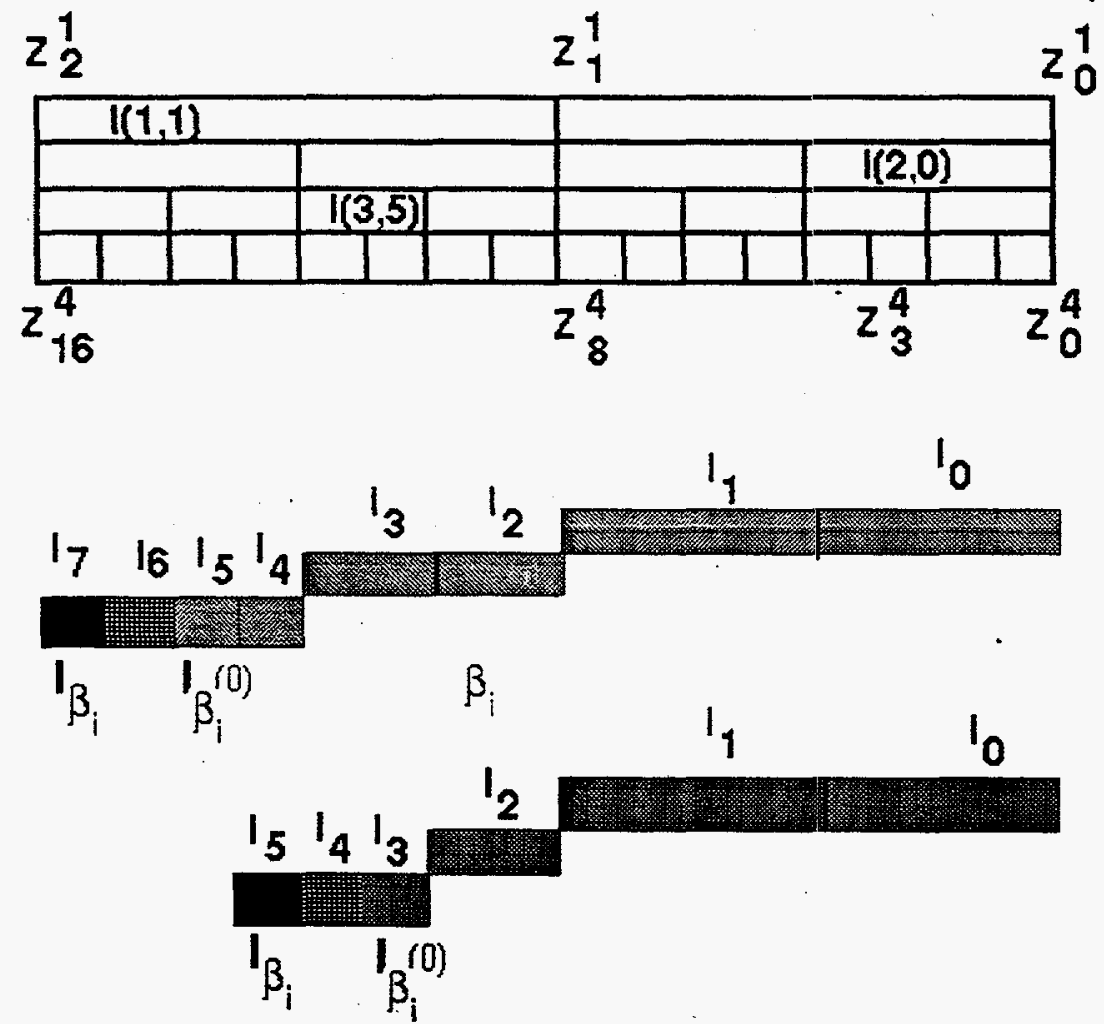

source

interval

direct

calculation

polynomial approximation

Figure 1: Illustration of decomposition for $L=4$, and two different choices for $\beta_{i}$. 